\title{
Consumo de hojas por herbívoros en manglares del estuario del Río Dagua, Costa Pacífica Colombiana
}

\author{
Isabel C. Romero ${ }^{1}$, Jaime R. Cantera K. ${ }^{2}$ \& Enrique J. Peña S. ${ }^{3}$ \\ Departamento de Biología, Universidad del Valle, AA 25360, Cali, Colombia. 1iromero@usc.edu \\ jcantera@univalle.edu.co \\ enripena@univalle.edu.co
}

Recibido 05-VI-2002. C Corregido 17-VI-2005. Aceptado 09-VIII-2006.

\begin{abstract}
Leaf consumption by herbivores in mangroves of the Dagua river estuary, Pacific coast of Colombia. Herbivore leaf consumption of various mangrove species in relation to environmental factors and leaf hardness were studied in the Dagua river estuary, Colombia. Leaf consumption and damage were assessed by measuring the percentage of area attacked by herbivores, distinguishing between consumption and damage. The species that suffered the highest consumption, such as Avicennia germinans (Avicenniaceae) and Laguncularia racemosa (Combretaceae), had softer leaves and less herbivore species when compared with Rhizophora spp. (Rhizophoraceae) and Pelliciera rhizophorae (Theaceae). The abundance and diversity of leaf grazing and its variability among mangrove species in the Dagua River estuary, show the importance of the trophic dynamics of live vegetable matter, in spite of their relatively low contribution to removing organic matter. Rev. Biol. Trop. 54 (4): 1205-1214. Epub 2006 Dec. 15.
\end{abstract}

Key words: herbivory, leaf grazing, mangrove, estuaries, Colombia.

La estructura trófica de los bosques de manglar tiene su base en las transformaciones de materia orgánica originada por la producción de hojarasca de los árboles de mangle (Prahl et al. 1990). Estas hojas se encuentran parcialmente consumidas por herbívoros dependiendo de diferentes factores físicos o procesos biológicos. De esta manera, la acción de los herbívoros puede regular la productividad primaria y ejercer un papel fundamental en las interacciones tróficas de los bosques de manglar (Robertson 1991).

Estudios anteriores han demostrado que factores como el efecto de los nutrientes (Onuf et al. 1977), la distancia al continente (Farnsworth y Ellison 1991) y la intervención humana (Johnstone 1981, Lacerda et al. 1986) pueden incrementar la tasa de consumo por herbívoros (Blanchard y Prado 1995). Muy pocos trabajos han tratado de establecer las características propias de cada especie de herbívoro, sus diferencias y el tipo de destrucción que causan sobre el material vegetal. Además la mayoría de ellos han sido realizados en regiones diferentes al Pacífico suramericano y en ambientes diferentes a los estuarios (Onuf et al. 1977, Johnstone 1981, Lacerda et al. 1986, Robertson y Duke 1987, Farnsworth y Ellison 1991, Anderson y Lee 1995, Feller 1995, Stowe 1995). En los manglares del Pacífico tropical americano, y en particular en la costa colombiana, existe poco conocimiento sobre el consumo foliar y sus características. Se han observado algunas larvas de insectos (Sphingidae, Ascolapha odorata, Phoebis sp.), cangrejos (Aratus pisonii, Sesarma spp.) y venados (Odocoileus sp.) consumiendo hojas de mangle (Prahl et al. 1990) pero sólo se ha cuantificado el consumo foliar por herbívoros en Rhizophora sp. en la Bahía de Málaga (Romero y Cantera 1996).

Este trabajo presenta de manera descriptiva varios aspectos sobre el tipo de destrucción 
o daño que los herbívoros causan en las hojas, las tasas de consumo en diferentes zonas a lo largo del estuario con diferentes características estuarinas y las actividades antropogénicas, como la tala de los mangles en un bosque ribereño de manglar del estuario del río Dagua.

\section{MATERIALES Y MÉTODOS}

El presente estudio se llevó a cabo en el estuario del río Dagua $\left(3^{\circ} 51^{\prime} \mathrm{N}, 7^{\circ} 4^{\prime} \mathrm{W}\right.$ ), localizado en la bahía de Buenaventura (Fig. 1A) La bahía está localizada en una zona altamente lluviosa durante todo el año (6 000$8000 \mathrm{~mm}$ ) con dos picos un en abril-mayo y otro entre septiembre y noviembre. Las mareas son semidiurnas, regulares con una amplitud máxima de 3.7 m (Cantera y Blanco 2001). El estuario del río Dagua se dividió, con el fin de ubicar las estaciones de muestreo, en tres zonas (cabecera, estuario propiamente dicho y desembocadura) con diferencias en sus características hidrológicas (Fig. 1B). Se identificaron los árboles de mangle predominantes: Rhizophora sp. (Rhizophoraceae), Avicennia germinans (L.) (Avicenniaceae), Laguncularia racemosa (L.) Gaertn 1807 (Combretaceae) y Pelliciera rhizophorae Triana \& Planchon 1862 (Theaceae). En las tres zonas se ubicaron cinco estaciones cubriendo áreas de manglares en buen estado y áreas con diferentes grados de intervención por tala (Cantera 1991, Cantera y Blanco 2001). Las características de las cinco estaciones se presentan en el Cuadro 1.

En cada estación, se seleccionaron al azar hojas maduras de cinco árboles adultos de cada especie que estuvieran ubicadas a una altura media en el dosel con exposición lumínica intermedia. En cada árbol, se cortó la terminación de una rama con 30-40 hojas maduras, de las cuales fueron seleccionadas al azar 20 hojas, limpiadas de sedimentos finos acumulados sobre ellas y almacenadas en bolsas de polietileno para su posterior análisis en el laboratorio. Se dibujaron en papel todas las señales de consumo por herbívoros. El porcentaje de área foliar consumida se calculó midiendo el área de cada señal y el área total de la hoja utilizando un sistema digital de tratamiento de imágenes. También se calculó el área foliar consumida como la suma de todas las señales observadas en cada hoja y se determinó la frecuencia de hojas con señales de haber sido consumidas (hojas atacadas por herbívoros/total hojas). En las hojas con el margen removido, el área se determinó reconstruyéndolo visualmente a partir de la porción intacta de la hoja (Farnsworth y Ellison 1991). El consumo por herbívoros se expresó en términos de proporciones para minimizar el error asociado a la expansión de los huecos durante el crecimiento de las hojas (Lowman 1984). Para las comparaciones entre especies de mangle, el consumo de hojas se
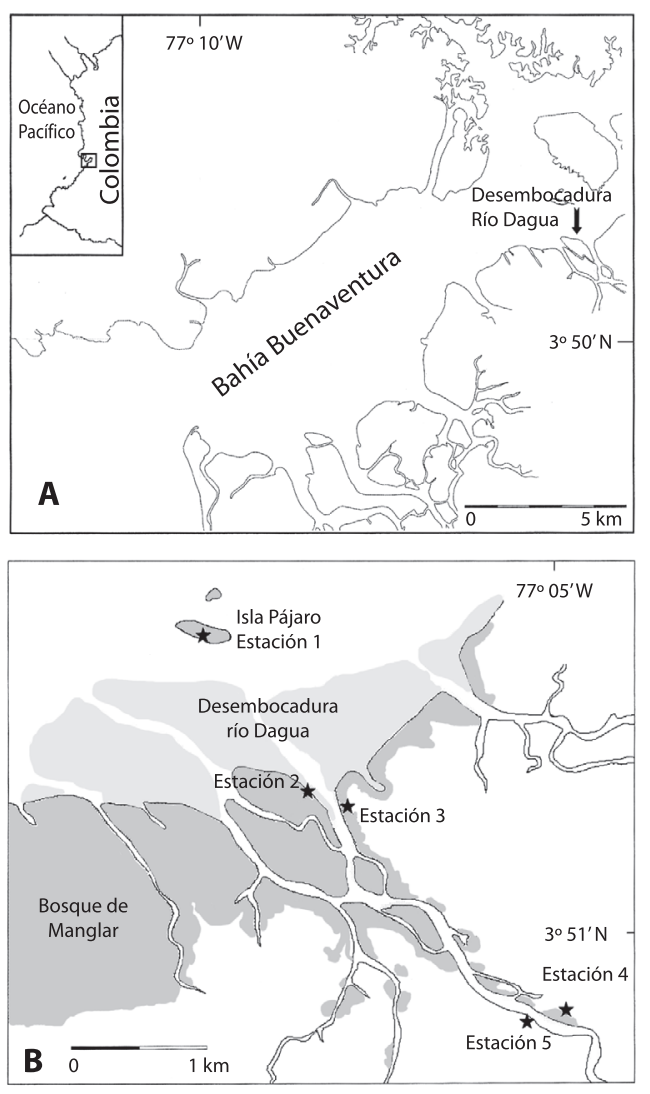

Fig. 1. Localización del Estuario del río Dagua (A) y de las zonas de estudio (B) en las zonas del estuario

Fig. 1. Location of Dagua river estuary (A) and of study sites (B) at each estuarine zone. 
CUADRO 1

Características generales de las estaciones donde se midió el consumo por herbívoros en el estuario del Río Dagua

TABLE 1

General characteristics of the sample stations for measurement of herbivory in the estuary of Dagua River

\begin{tabular}{|c|c|c|c|c|c|}
\hline ESTACIÓN & LOCALIZACIÓN & $\begin{array}{l}\text { SALINIDAD } \\
\text { (ups) }\end{array}$ & $\begin{array}{c}\text { ESTADO DE } \\
\text { DESARROLLO }\end{array}$ & $\begin{array}{c}\text { PARÁMETRO } \\
\text { ESTRUCTURALES }\end{array}$ & $\begin{array}{c}\text { GÉNERO } \\
\text { DOMINANTE }\end{array}$ \\
\hline $1(\mathrm{E}-\mathrm{NT})$ & $\begin{array}{l}\text { Isla Pájaro Zona } \\
\text { externa }\end{array}$ & $11.2-17.5$ & Buen desarrollo & $\begin{array}{l}\text { Altura máxima } 15.5 \mathrm{~m} \\
\text { Área basal } 0.62 \mathrm{~m}^{2} / 0.1 \mathrm{ha} \\
\text { Densidad } 112 \text { árboles } / 0.1 \mathrm{ha}\end{array}$ & Rhizophora \\
\hline $2(\mathrm{M}-\mathrm{NT})$ & $\begin{array}{l}\text { Boca del Estuario } \\
\text { Zona externa }\end{array}$ & $4,$. & Buen desarrollo & $\begin{array}{l}\text { Altura máxima } 13.7 \mathrm{~m} \\
\text { Área basal } 2.3 \mathrm{~m}^{2} / 0.1 \text { ha } \\
\text { Densidad } 84 \text { árboles } / 0.1 \text { ha }\end{array}$ & P. rhizophorae \\
\hline $3(\mathrm{M}-\mathrm{T})$ & $\begin{array}{l}\text { Boca del Estuario } \\
\text { Zona externa }\end{array}$ & $4.0-20.0$ & $\begin{array}{l}\text { Muy intervenido } \\
\text { con tala }\end{array}$ & $\begin{array}{l}\text { Altura máxima } 13.7 \mathrm{~m} \\
\text { Área basal } 2.3 \mathrm{~m}^{2} / 0.1 \text { ha } \\
\text { Densidad } 84 \text { árboles } / 0.1 \text { ha }\end{array}$ & A. aureum \\
\hline 4 (I-NT) & $\begin{array}{l}3 \mathrm{Km} \text { aguas arriba } \\
\text { Zona interna }\end{array}$ & $0-9.0$ & Buen desarrollo & $\begin{array}{l}\text { Altura máxima } 6.7 \mathrm{~m} \\
\text { Área basal } 9.8 \mathrm{~m}^{2} / 0.1 \text { ha } \\
\text { Densidad } 3.6 \text { árboles } / 0.1 \mathrm{ha}\end{array}$ & Rhizophora \\
\hline $5(\mathrm{I}-\mathrm{T})$ & $\begin{array}{l}3 \mathrm{Km} \text { aguas arriba } \\
\text { Zona interna }\end{array}$ & $0-9.0$ & $\begin{array}{l}\text { Muy intervenido } \\
\text { con tala }\end{array}$ & $\begin{array}{l}\text { Altura máxima } 6.7 \mathrm{~m} \\
\text { Área basal } 9.8 \mathrm{~m}^{2} / 0.1 \text { ha } \\
\text { Densidad } 3.6 \text { árboles } / 0.1 \text { ha }\end{array}$ & $\begin{array}{l}\text { Rhizophora } \\
\text { Acrostrichium } \\
\text { cultivos } \\
\text { agrícolas }\end{array}$ \\
\hline
\end{tabular}

calculó como el porcentaje promedio por hoja de área con consumo por herbívoros (Williams y Abbott 1991). Las hojas se pesaron para estimar el peso húmedo por unidad de área $\left(\mathrm{g} / \mathrm{cm}^{2}\right)$ como indicativo de la dureza de las hojas (Feller 1995), la cual puede afectar los niveles de consumo foliar por herbívoros (Coley 1987). Los porcentajes de consumo por herbívoros se compararon entre las especies, también entre las estaciones de muestreo para cada especie, utilizando análisis de varianza de una vía con número desigual de repeticiones. Para determinar el efecto de los factores ambientales sobre los porcentajes calculados, se utilizó el análisis de varianza de dos vías (ANOVA factorial) con los factores salinidad y condición del bosque. Para los análisis con diferencias significativas se realizaron comparaciones múltiples de Newman-Keuls (Zar 1996).

\section{RESULTADOS}

Patrones generales: Se encontraron 12 formas o señales de consumo de hojas por herbívoros en las hojas de mangle, las cuales fueron clasificadas en general como "daño estructural", si el daño ocasionaba pérdida total o parcial del material foliar, o como "daño superficial", si presentaban manchas de tejido necrótico, hongos u otros organismos y otras marcas que representan daño de la superficie del tejido foliar dejado por los herbívoros, al alimentarse de la savia de las hojas (Cuadro 2). Las señales más comunes de ataque de herbívoros a las hojas se presentan en la Fig. 2. El $94.1 \%$ del área foliar utilizada por los herbívoros es removida por organismos masticadores. Los raspados en la epidermis de las hojas son ocasionados por insectos del orden Thysanoptera (Familia: Phlaeothripidae), moluscos gasterópodos de la familia: Littorinidae y el cangrejo Aratus pisonii (Familia: Grapsidae).Los huecos pueden ser ocasionados por larvas de coleópteros (Curculionidae), lepidópteros (Pyralidae), grillos (Tettigoniidae, Gryllidae) y los cangrejos A. pisonii y Goniopsis pulcra Lockington, 1876. Las manchas de tejido foliar son realizadas por insectos succionadores del orden Hemiptera (Aphididae), por la descomposición 
CUADRO 2

Tipos de daños en las hojas como consecuencia de la acción de los herbivoros observados en las especies de mangle en el estuario del río Dagua

TABLE 2

Herbivore consumption and damage signal types observed on mangrove leaves from Dagua river estuary

\begin{tabular}{|c|c|c|}
\hline Tipo de daño o señal & & Descripción \\
\hline \multicolumn{3}{|c|}{ Pérdida parcial de la superficie de la hoja } \\
\hline Raspado & $(50.4 \%)$ & $\begin{array}{l}\text { Pérdida de la capa superficial (epidermis) de las hojas sin ningún patrón excepto } \\
\text { en } L \text {. racemosa en donde se presenta alineado en el borde. }\end{array}$ \\
\hline Huecos I & $(34.4 \%)$ & Perforaciones o aberturas en la lámina de las hojas. \\
\hline Huecos II & $(62 . . \%)$ & $\begin{array}{l}\text { Pérdida de materia! foliar en e! margen de las hojas que se pueden prolongar } \\
\text { hacia el interior de la lámina. En L. racemosa se presentan a ambos lados de las } \\
\text { hojas desde el margen hacia el interior. }\end{array}$ \\
\hline \multicolumn{3}{|l|}{ Daños superficiales } \\
\hline Tejido necrótico & $(12.0 \%)$ & $\begin{array}{l}\text { Manchas de color negro en forma de tubérculos ovalados o hundimientos en la } \\
\text { vena central y la lámina de las hojas. También se pueden observar como líneas } \\
\text { oscuras en la superficie de la hoja. }\end{array}$ \\
\hline Hongo I & $(11.6 \%)$ & $\begin{array}{l}\text { Mancha oscura redondeada u ovalada en la lámina de la hoja. Puede ser la } \\
\text { enfermedad conocida como Antracnosis. }\end{array}$ \\
\hline Hongo II & $(4.2 \%)$ & $\begin{array}{l}\text { Grandes manchas de color pardo rojizo sobre el margen de la hoja, sin ninguna } \\
\text { forma definida. }\end{array}$ \\
\hline Hongo III & $(1.8 \%)$ & $\begin{array}{l}\text { Mancha compuesta por una pelusa fina de color marrón claro con forma redon- } \\
\text { deada. Puede ser la enfermedad conocida como Mildeo. }\end{array}$ \\
\hline Escamas & $(0.4 \%)$ & $\begin{array}{l}\text { Mancha café oscuro sobre el haz de la hoja, cubren pequeñas porciones de la } \\
\text { lámina. }\end{array}$ \\
\hline Agallas & $(3.2 \%)$ & $\begin{array}{l}\text { En las venas o en la lámina de la hoja aisladas o en grupo. Cuando crecen sobre } \\
\text { las venas deforman la hoja. }\end{array}$ \\
\hline Minas & $(1.6 \%)$ & $\begin{array}{l}\text { Con forma lineal o sin un patrón definido sobre la lámina de las hojas Cuando } \\
\text { son muy grandes deforman las hojas. }\end{array}$ \\
\hline Huevos & $(1.3 \%)$ & $\begin{array}{l}\text { Ubicados en la vena central, con ellos se observan pequeñas cortaduras en el } \\
\text { envés de la hoja. }\end{array}$ \\
\hline Marcas & $(1.3 \%)$ & $\begin{array}{l}\text { Cicatrices ovaladas observadas en la vena central de las hojas o como líneas } \\
\text { transversales sobre la lámina. }\end{array}$ \\
\hline
\end{tabular}

En paréntesis: frecuencia (\%) de cada tipo de señales de ataque sobre las hojas ( $\mathrm{N}=1730)$.

In parenthesis: frequency (\%) of each type of attack signals $(\mathrm{N}=1730)$.

de la epidermis por hongos, agallas, minas o huevos ocasionados por el orden Díptera (Agromyzidae).

Los huecos y los raspados son las señales de ataque más frecuentes $(96.7 \%$ y $50.4 \%$ respectivamente). Los hongos y los tejidos necróticos son los daños superficiales más importantes (Cuadro 2). El $54.3 \%$ de las hojas presentan por lo menos dos tipos de señales de consumo observándose en algunos casos, como en L. racemosa, hasta siete tipos diferentes en una misma hoja. El $84.9 \%$ del total de las hojas han sido atacadas por herbívoros. A pesar de esto, la mayoría de las hojas no presentan más del $0.5 \%$ de su área dañada por herbívoros.

Comparación entre especies: Las especies de mangle estudiadas presentaron diferen- 

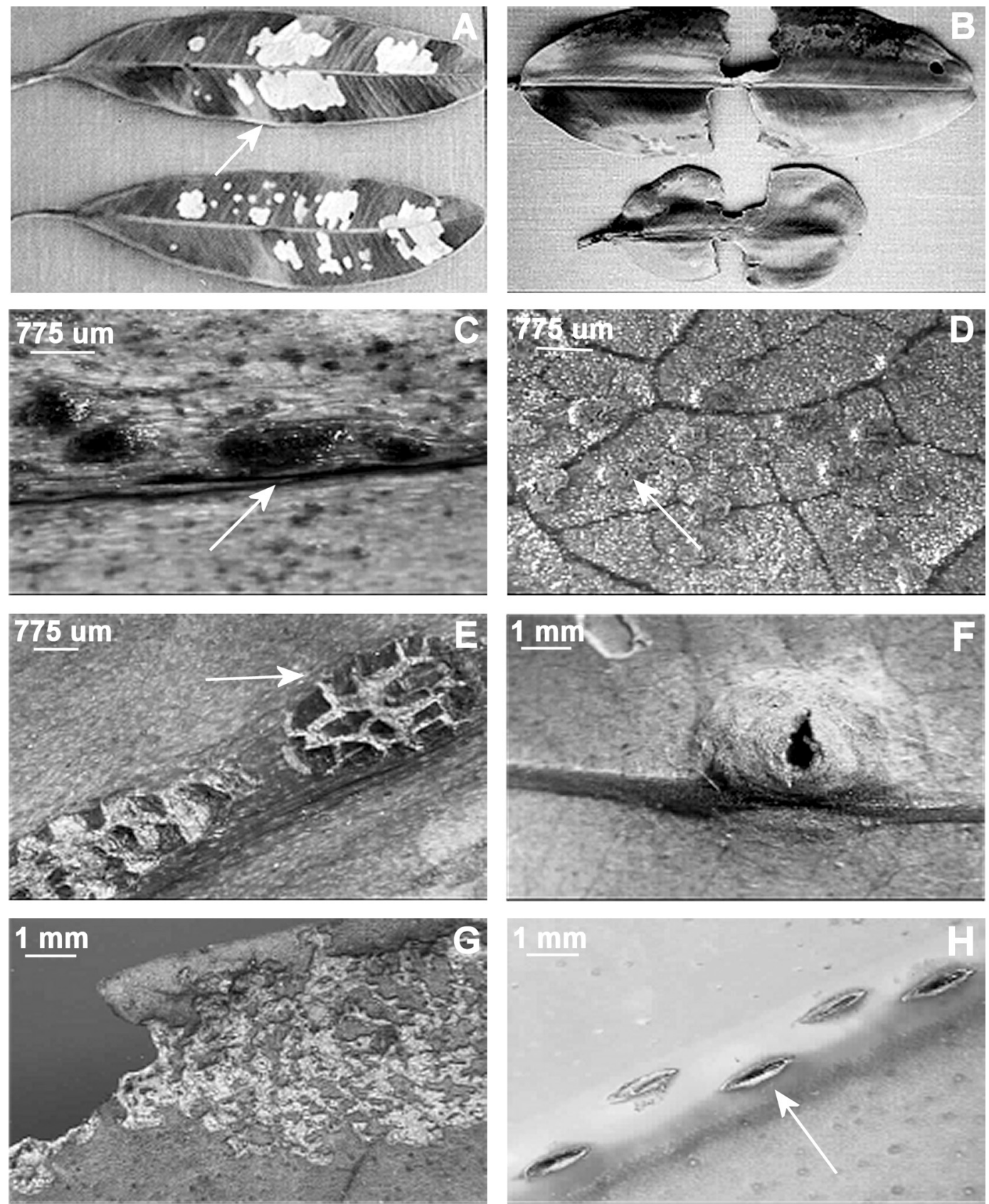

Fig. 2. Tipos de daños causados por herbívoros en las hojas de las especies de mangle del estuario del río Dagua. A. raspado en A. germinans; B. huecos tipo II en L. racemosa; C. manchas de tejido necrótico en L. racemosa; D. hongos tipo III en A. germinans; E. marcas en L. racemosa; F. agallas en A. germinans; G. minas en L. racemosa; H. huevos de insectos en Rhizophora spp.

Fig. 2. Types of herbivory on mangrove leaves from Dagua river estuary. A. leaf scrapes in A. germinans; B. holes type II in L. racemosa; C. spots of necrotic tissue in L. racemosa; D. type III fungi in A. germinans; E. scars in L. racemosa; F. galls in A. germinans; G. mines in L. racemosa; H. insect eggs in Rhizophora spp. 
cias significativas en los niveles de consumo por herbívoros $(\mathrm{p}<0.001)$, con los valores promedios más altos en $A$. germinans (4.9\%) y $L$. racemosa $(4.7 \%)$ y los más bajos en Rhizophora sp. (2.3\%) y P. rhizophorae $(2.1 \%)$. A pesar de los bajos porcentajes encontrados para cada especie, se encontró que en todas las especies las hojas son muy utilizadas por los herbívoros, (el $77 \%$ de las hojas presentan algún tipo de señales de consumo por herbívoros (Cuadro 3). Los huecos presentan los porcentajes más altos en todas las especies, menos en $A$. germinans en donde los raspados son los más importantes (Fig. 3). Entre los daños superficiales, los hongos tienen los porcentajes más altos en todas las especies con excepción de $L$. racemosa. Aunque la mayoría de las señales se encuentran en varias especies de mangle, algunas pueden ser específicos a una especie de mangle, como hongo III en A. germinans, escamas y hongos I en $P$. rhizophorae y presencia de huevos en Rhizophora. La dureza de las hojas $\left(\mathrm{g} / \mathrm{cm}^{2}\right)$ también presentó diferencias significativas entre las especies de mangle $(\mathrm{p}<0.001)$. Las hojas con mayor peso por unidad de área es Rhizophora $(0.043 \pm 0.0002)$ seguido de $P$. rhizophorae $(0.039 \pm 0.0003), L$. racemosa $(0.035 \pm 0.0004)$ y por último, A. germinans $(0.035 \pm 0.0004)$.

Variación espacial del consumo en el estuario: El consumo por herbívoros total, expresado como el porcentaje promedio de área foliar con señales de ataque por herbívoros de todas las muestras de hojas es variable dentro del área de estudio. Rhizophora sp. y P. rhizophorae presentaron diferencias significativas

CUADRO 3

Porcentaje de área foliar con consumo por herbivoros $(\bar{x} \pm$ error estándar)

TABLE 3

Percentage of foliar area affected by herbivory $(\bar{x} \pm$ standard error $)$

\begin{tabular}{|c|c|c|c|c|c|c|}
\hline \multicolumn{2}{|c|}{ Estaciones } & \multicolumn{4}{|c|}{ Especies } & \multirow[b]{2}{*}{ Total } \\
\hline Zonas & $\begin{array}{c}\text { Condición } \\
\text { bosques }\end{array}$ & Rhizophora & P. rhizophorae & A. germinans & L. racemosa & \\
\hline $1 \mathrm{E}-\mathrm{NT}$ & No talado & $\begin{array}{c}3.22 \pm 0.55^{\mathrm{a}} \\
(\mathrm{N}=100)\end{array}$ & $\begin{array}{c}2.64 \pm 0.48^{\mathrm{a}} \\
(\mathrm{N}=100)\end{array}$ & ---------- & --------- & $\begin{array}{c}2.93 \pm 0.37^{\mathrm{a}} \\
(\mathrm{N}=200)\end{array}$ \\
\hline $2 \mathrm{M}-\mathrm{NT}$ & No talado & $\begin{array}{c}3.23 \pm 0.49^{\mathrm{a}} \\
(\mathrm{N}=100)\end{array}$ & $\begin{array}{c}2.48 \pm 0.55^{\mathrm{a}} \\
(\mathrm{N}=100)\end{array}$ & $\begin{array}{c}4.51 \pm 0.60^{\mathrm{a}} \\
(\mathrm{N}=100)\end{array}$ & $\begin{array}{c}4.27 \pm 0.52^{\mathrm{a}} \\
(\mathrm{N}=100)\end{array}$ & $\begin{array}{c}3.62 \pm 0.27^{\mathrm{b}} \\
(\mathrm{N}=400)\end{array}$ \\
\hline $3 \mathrm{M}-\mathrm{T}$ & Talado & $\begin{array}{c}2.43 \pm 0.22^{\mathrm{a}} \\
(\mathrm{N}=100)\end{array}$ & $\begin{array}{c}2.97 \pm 0.48^{\mathrm{a}} \\
(\mathrm{N}=100)\end{array}$ & $\begin{array}{c}3.96 \pm 0.47^{\mathrm{a}} \\
(\mathrm{N}=100)\end{array}$ & $\begin{array}{c}8.10 \pm 1.20^{b} \\
(\mathrm{~N}=31)\end{array}$ & $\begin{array}{c}3.59 \pm 0.25^{\mathrm{b}} \\
(\mathrm{N}=331)\end{array}$ \\
\hline $4 \mathrm{I}-\mathrm{NT}$ & No talado & $\begin{array}{l}1.22 \pm 0.21^{\mathrm{b}} \\
(\mathrm{N}=100)\end{array}$ & $\begin{array}{c}0.86 \pm 0.14^{\mathrm{b}} \\
(\mathrm{N}=99)\end{array}$ & $\begin{array}{c}3.87 \pm 0.49^{\mathrm{a}} \\
(\mathrm{N}=100)\end{array}$ & $\begin{array}{c}3.82 \pm 0.61^{\mathrm{a}} \\
(\mathrm{N}=100)\end{array}$ & $\begin{array}{c}2.44 \pm 0.22^{\mathrm{c}} \\
(\mathrm{N}=399)\end{array}$ \\
\hline $5(\mathrm{I}-\mathrm{T})$ & Talado & $\begin{array}{c}1.43 \pm 0.28^{\mathrm{b}} \\
(\mathrm{N}=100)\end{array}$ & $\begin{array}{c}1.34 \pm 0.23^{b} \\
(\mathrm{~N}=100)\end{array}$ & $\begin{array}{c}7.25 \pm 0.87^{b} \\
(\mathrm{~N}=100)\end{array}$ & $\begin{array}{c}5.01 \pm 0.50^{\mathrm{a}} \\
(\mathrm{N}=100)\end{array}$ & $\begin{array}{c}3.75 \pm 0.29^{\mathrm{b}} \\
(\mathrm{N}=400)\end{array}$ \\
\hline \multicolumn{2}{|c|}{$\begin{array}{l}\text { Frecuencia de hojas } \\
\text { con herbivoría (\%) }\end{array}$} & 78.0 & 77.3 & 91.0 & 99.1 & \\
\hline \multicolumn{2}{|c|}{ \% Herbivoría } & $2.30 \pm 0.17$ & $2.06 \pm 0.19$ & $4.90 \pm 0.32$ & $4.72 \pm 0.31$ & \\
\hline \multicolumn{2}{|c|}{ Rango \% Herbivoría } & $0.01-47.38$ & $0.02-40.68$ & $0.03-37.16$ & $0.07-45.20$ & \\
\hline
\end{tabular}

Los promedios por estación para cada especie con diferencias significativas están señalados con diferentes letras (ANOVA, $\mathrm{P}<0.05)$. --- Porcentajes no medidos. Significant differences for each sampling site and mangrove species are shown with different letters (ANOVA, $\mathrm{P}<0.05$ ). ---- Percentage not measured. 

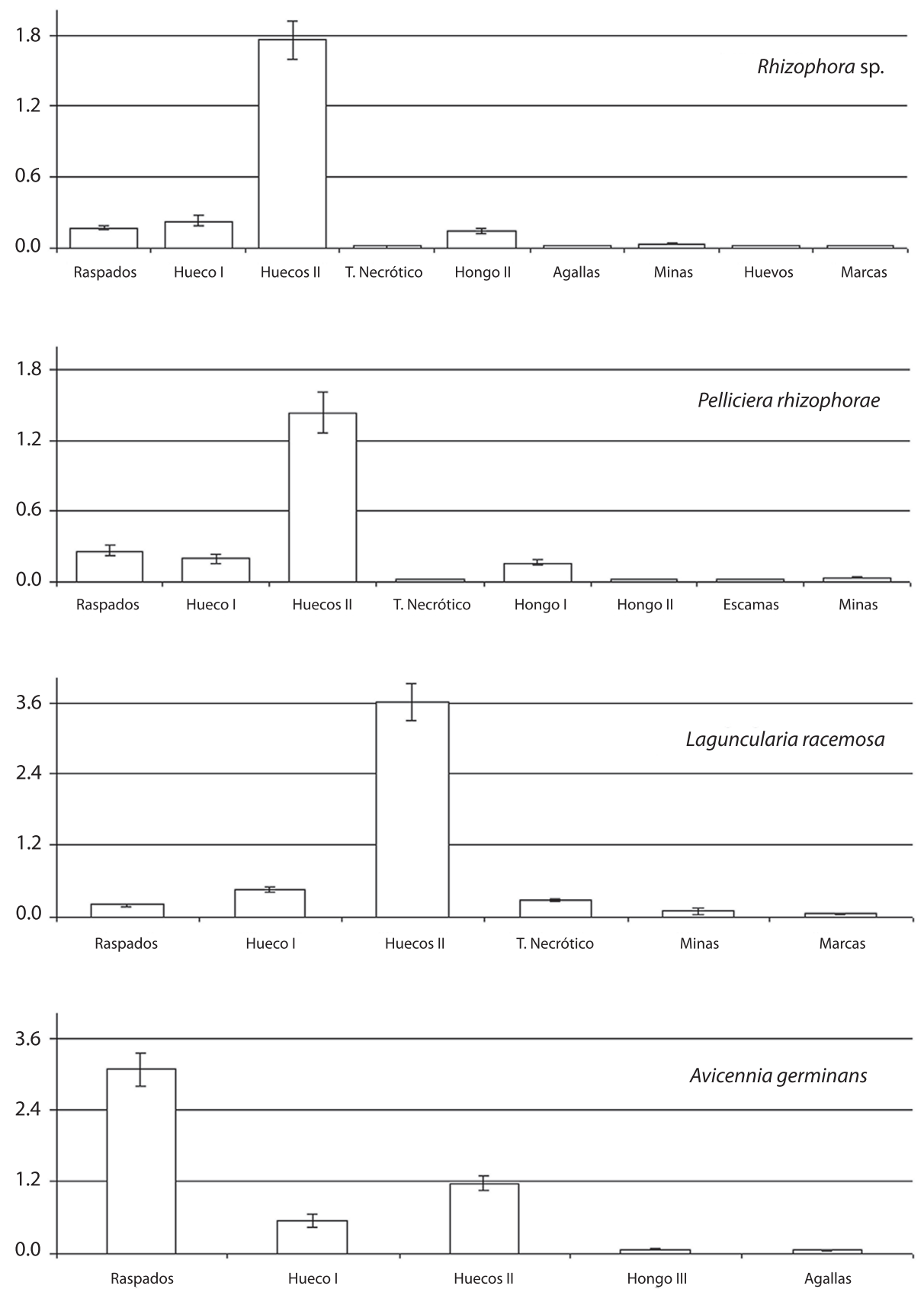

Fig. 3. Distribución de los tipos de consumo por herbívoros en las hojas de las especies de mangle en el estuario del río Dagua. Los valores expresan el porcentaje de área foliar consumida ( $\overline{\mathrm{x}} \pm$ error estándar), mostrando la importancia relativa de cada tipo de consumo en las hojas de mangle.

Fig. 3. Distribution of herbivory types on the leaves of mangrove species from Dagua river estuary. Values are expressed as the percent of foliar herbivory $(\overline{\mathrm{x}} \pm \mathrm{SE})$. 
entre las zonas estuarinas con los promedios más altos en las zonas media y externa (Cuadro 3). A. germinans solo mostró diferencias significativas con el efecto combinado del consumo y la localización en el estuario. L. racemosa presentó diferencias significativas entre la localización, las características de los bosques y la interacción de estos dos factores $(\mathrm{p}<0.05)$.

El análisis de la importancia relativa de los factores sobre el consumo por herbívoros mostró que para todas las especies estudiadas, la condición del bosque, el grado de intervención y la zona del manglar donde se encuentran los árboles son los principales factores que determinan la tasa y el tipo de consumo que sufren las especies, aunque la salinidad puede ser otro factor que explica la distribución de los daños en las hojas a lo largo del estuario.

\section{DISCUSIÓN}

El consumo de hojas de mangles en el estuario del río Dagua se caracterizó por la alta variabilidad. Las hojas de estas especies fueron aprovechadas por una gran variedad de organismos herbívoros que consumieron el material foliar vivo, exportando este material a niveles superiores de la red trófica estuarina (Farnsworth y Ellison 1991, Stowe 1995). Aunque se ha propuesto que un modelo de estudio basado en los tipos de daños puede describir los consumidores de hojas de manglar (Smith et al. 1989), es necesario tener en cuenta que la asociación entre herbívoros y las estrategias de alimentación pueden depender de las etapas de su ciclo de vida. Por ejemplo, los raspados en la epidermis de las hojas efectuados por $A$. pisonii pueden permanecer intactos hasta seis semanas después, cuando se oscurecen por el crecimiento de hongos (Beever et al. 1979).

Las hojas de los mangles del estuario del río Dagua son fuertemente atacadas por herbívoros, a pesar de que el área promedio de cada hoja consumida por herbívoros es inferior al $5 \%$. El consumo directo de tejidos presenta un promedio de $3.3 \%$ y los daños superficiales como hongos y agallas representan el
$0.2 \%$. Estos porcentajes, aunque bajos, son comparables con otros trabajos realizados en el ecosistema de manglar sobre consumo por herbívoros y daños foliares (Johnstone 1981, Lowman 1984, Lacerda et al. 1986, Robertson y Duke 1987, Romero y Cantera 1996). Los herbívoros han sido considerados como importantes en el funcionamiento y regulación del ecosistema de bosque, al poder alterar el flujo de materia orgánica (Anderson y Lee 1995) y afectar la estructura y patrones de sucesión de los manglares (Robertson et al. 1990).

Es probable que una variedad de factores hayan sido los responsables de las diferencias observadas entre las especies de mangle, como la dureza física y el contenido químico de las hojas (Colley 1987, Feller 1995). Las especies con las hojas más duras, presentaron los porcentajes promedios de consumo más bajos, mientras que las hojas con menos dureza tuvieron los porcentajes más altos ( $L$. racemo$s a$ y $A$. germinans). Esta misma tendencia ha sido observada en otros manglares del mundo (Robertson y Duke 1987). El mayor porcentaje promedio de consumo encontrado en $A$. germinans puede explicarse por la presencia de cristales de sal en la superficie de las hojas que inhiben el ataque de ciertos herbívoros (Newbery 1980, Farnsworth y Ellison 1991).

La variación encontrada en el consumo foliar por herbívoros entre las especies de mangle, puede estar relacionada con el desarrollo foliar, el cual depende del desarrollo estructural de los bosques. Así, cada especie de mangle presenta adaptaciones particulares para soportar las condiciones ambientales como cambios en la salinidad del agua (Soto y Corrales 1987), en la temperatura, la iluminación y la composición faunística. Finalmente, las condiciones de intervención humana, como la tala de árboles, pudieron afectar también, el consumo por herbívoros. En la zona interna del estuario se presentaron diferencias importantes entre el bosque talado $(3.7 \%)$ y el no talado $(2.4 \%)$. El patrón de distribución espacial de los tipos de daños con respecto a los factores ambientales del estuario varió drásticamente entre las especies, lo cual hace difícil hacer 
generalizaciones. Al parecer, las especies de mangle no comparten todos los mismos herbívoros (Farnsworth y Ellison 1991) aunque es conocido que $A$. pisonii puede comer hojas de $R$. mangle, de A. germinans y L. racemosa (Beever et al. 1979). La alta variabilidad de las modalidades del consumo foliar por herbívoros en el estuario del río Dagua y la abundancia del material foliar afectado por la acción de estos organismos permite afirmar que, a pesar del bajo porcentaje promedio de área foliar (inferior al $10 \%$ ), los herbívoros ejercen un papel importante en la red trófica del sistema estuarino.

\section{AGRADECIMIENTOS}

Esta investigación es parte del Proyecto Transferencia de Materia y Energía en el Estuario del río Dagua, financiado por el Instituto Colombiano para el Desarrollo de la Ciencia y la Tecnología Francisco José de Caldas (COLCIENCIAS, proyecto No. 110609-328-97) y por la Universidad del Valle. Se agradece el apoyo logístico ofrecido por la Sección de Biología Marina de la Universidad del Valle, y la participación de G. Toro, E. Rodríguez, P. Tabares, R. Neira y C. Fernández en diversos aspectos de este trabajo.

\section{RESUMEN}

Se estudió el consumo foliar por herbívoros en hojas de varias especies de mangle con relación a los factores ambientales y la dureza de las hojas en el estuario del río Dagua. La intensidad del consumo o de los daños producidos en las hojas se cuantificó determinando el porcentaje de área foliar afectado por herbívoros separando las distintas señales de consumo o daño de las hojas. Las especies más consumidas, como A germinans y L. racemosa, presentan el menor número de tipos de huellas de daños y menor dureza que las especies menos consumidas como Rhizophora sp. y $P$. rhizophorae. La abundancia y diversidad de huellas de ataque por herbívoros y su variabilidad a lo largo del estuario del río Dagua, muestra la importancia de los procesos de consumo de tejido vegetal vivo en el bosque de manglar dentro de la red trófica del sistema estuarino.
Palabras clave: herbivoría, consumo de hojas, ramoneo, mangle, estuarios, Colombia.

\section{REFERENCIAS}

Anderson, C. \& S.Y. Lee. 1995. Defoliation of the mangrove Avicennia marina in Hong Kong: cause and consequences. Biotropica 27: 218-226.

Beever, J.W., D. Simberloff \& L. King. 1979. Herbivory and predation by the mangrove tree crab Aratus pisonii. Oecologia 43: 317-328.

Blanchard, J. \& G. Prado. 1995. Natural regeneration of Rhizophora mangle in strip clear-cuts in Northwest Ecuador. Biotropica 27: 160-167.

Cantera, J.R. 1991. Etude structurale des mangroves et des peuplements littoraux des deux baies du pacifique colombien (Málaga et Buenaventura). Rapport avec les conditions du milieu et les perturbations anthropiques. Thèse d'Etat Sciences. Université d'Aix-Marseille II. Marsella, Francia. 429 p.

Cantera, J.R. \& J.F. Blanco. 2001. The estuary ecosystem of Buenaventura Bay, Colombia, p. 265-280. In U. Seeliger \& B. Kjerfve (eds.). Coastal Marine Ecosystems of Latin America, Ecological studies. Springer, Nueva York, EEUU.

Coley, P.D. 1987. Patrones en las defensas de las plantas: ¿porqué los herbívoros prefieren ciertas especies? Rev. Biol. Trop. 35: 151-164.

Farnsworth, E. \& A. Ellison. 1991. Patterns of herbivory in Belizean mangrove swamps. Biotropica 23: 555 567.

Feller, I.C. 1995. Effects of nutrients enrichment on growth and herbivory of dwarf red mangrove (Rhizophora mangle). Ecol. Monog. 65: 477-505.

Johnstone, I. 1981. Consumption of leaves by herbivores in mixed mangrove stands. Biotropica 13: 252-259.

Lacerda, L.D., D.V. Jose, C.E. de Rezende, M.C.F. Francisco, J.C. Wasserman \& J.C. Martins. 1986. Leaf chemical characteristics affecting herbivory in a new world mangrove forest. Biotropica 18: 350-355.

Lowman, M.D. 1984. An assessment of techniques for measuring herbivory: Is rainforest defoliation more intense than we thought? Biotropica 16: 264-268.

Newbery, D. 1980. Infestation of the coccid, Icerya seychellarum (Westw.) on the mangrove Avicennia 
marina (Forsk.) Vierh. on Aldabra Atoll, with special reference to tree age. Oecologia 45: 325-330.

Onuf, C., J. Teal \& I. Valiela. 1977. Interactions of nutrient, plant growth and herbivory in a mangrove ecosystem. Ecology 58: 514-526.

Prahl, H., J. Cantera \& R. Contreras. 1990. Manglares y hombres del Pacífico colombiano. Fondo FEN Colombia, Santa Fe de Bogotá, Bogotá, Colombia. $193 \mathrm{p}$.

Robertson, A. 1991. Plant-animal interactions and the structure and function of mangrove forest ecosystems. Aust. Jour. Ecol. 16: 433-443.

Robertson, A. \& N. Duke. 1987. Insect herbivory on mangrove leaves in North Queensland. Aust. Jour. Ecol. 12: $1-7$.

Robertson, A.I., R. Giddins \& T.J. Smith. 1990. Seed predation by insects in tropical mangrove forests: extent and effects on seed viability and the growth of seedlings. Oecologia 83: 213-219.
Romero, I. \& J. Cantera. 1996. Herbivoría y variación en el área foliar del género Rhizophora (Familia Rhizophoracea) en Bahía Málaga. In I Simposio Nacional de Biología. Universidad del Valle, Cali, Colombia.

Smith, T.J., H.T. Chan, C.C. McIvor \& M.B. Robblee. 1989. Comparisons of seeds predation in tropical tidal forests from three continents. Ecology 70: 146-151.

Soto, R. \& L.F. Corrales. 1987. Variación de algunas características foliares de Avicennia germinans (L.) L. (Avicenniaceae) en un gradiente climático y de salinidad. Rev. Biol. Trop. 35: 245-256.

Stowe, K.A. 1995. Intracrown distribution of herbivore damage on Laguncularia racemosa in a tidally influenced riparian habitat. Biotropica 27: 509-512.

Williams, M.R. \& I. Abbott. 1991. Quantifying average defoliation using leaf-level measurements. Ecology 72: $1510-1511$

Zar, J.H. 1996. Biostatistical Analysis. Prentice Hall, Nueva Jersey, EEUU. 662 p. 